\title{
Protein Microarrays for Diagnostic Assays
}

\author{
Hartmann, Michael ${ }^{1,2}$; Roeraade, Johan $^{2}$ and Joos, Thomas ${ }^{1}$ \\ NMI Reutlingen \\ Markwiesenstraße 55, 72770 Reutlingen
}

A protein microarray, sometimes referred to as a protein chip, allows quantifying a large number of target analytes from minimal samples, using immobilized antibodies as capture molecules. Immobilized proteins in a microarray allow screening for reactive antibodies in serum samples, providing a multiplex approach to identify protein-protein interactions, identifying substrates of protein kinases or identifying the targets of biologically active small molecules. The array is a piece of chemically modified glass on which different capture molecules have been immobilized in separate spots in rows and columns thus forming a microarray. Typically hundreds or thousands of spots, each containing a unique capture molecule, are employed allowing to test for multiple binding events within a single assay. The dimension of a spot is approximately $150 \mu \mathrm{m}$ and some thousand spots can be immobilized on an area of the size of a one-cent coin (figure 1A). This high degree of parallelization and miniaturization of protein microarrays reduces sample and reagent consumption. According to Roger Ekins' ambient analyte theory such miniaturized immunoassays are under certain circumstances even more sensitive than classical immunoassays [Ekins 1989].

Protein microarrays are fabricated by so called microarrayers in a computer controlled fashion. These instruments allow to deliver volumes in the sub-nanoliter scale by applying both contact or non-contact printing techniques (figure 1B). During the assay samples of interests are incubated on the protein microarray. Basically, antibody antigen recognition or protein protein interaction is the underlying principle of the reaction between an immobilized capture molecule and a protein target analyte present in the sample. Finally, these reactions are usually visualized by fluorescence labels which are either directly introduced to the sample molecules or indirectly by fluorescently labeled reporter molecules in a sandwich assay format. For the fluorescence detection tailored laser scanner instruments are used and signals are extracted from scanned images with suitable image processing software.

(A)

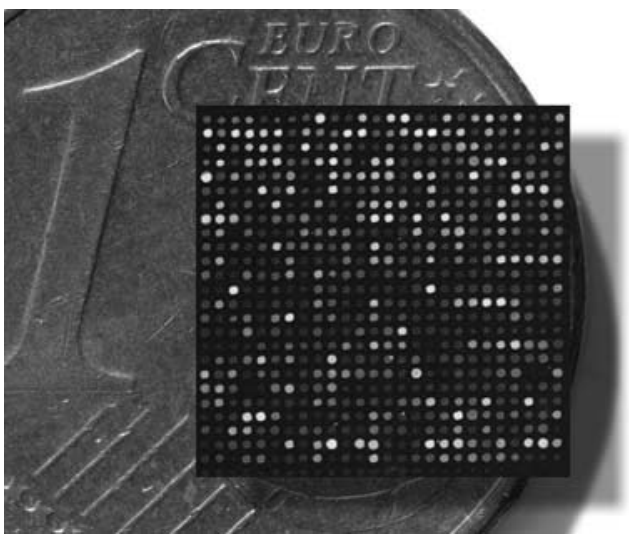

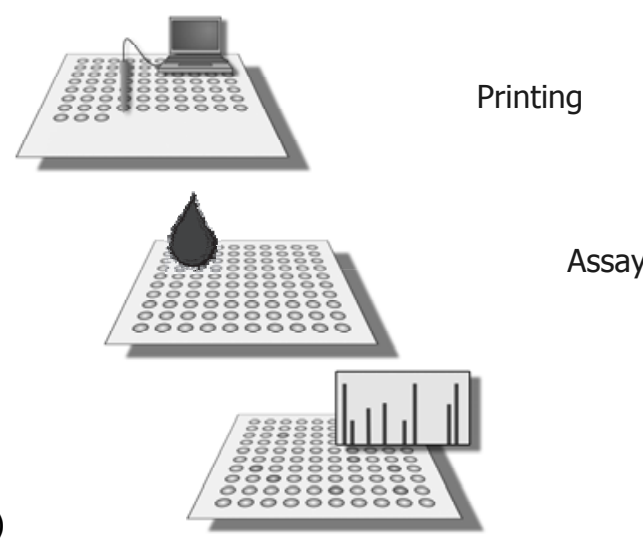

Readout

Figure 1: (A) Protein microarrays are highly parallelized and miniaturized solid phase assay systems consisting of many different capture molecules allowing testing for many different analytes within a single test. (B) Protein microarrays are fabricated in a computer controlled printing process by so called microarrayers. On a protein microarray a protein containing sample, e.g. body fluids, cell lysates, cell culture supernatants etc., is applied. The specific reaction of immobilized capture molecules and protein analytes is commonly indirectly reported by fluorescent readout with confocal laser scanning microscopy.

In general, protein microarray assay formats can be divided into forward-phase and reversed-phase microarrays. Forward-phase protein microarray assays, sometimes referred to as $\mu$ ELISA (Enzyme Linked Immunosorbent Assay), involve the immobilization of capture molecules and hence enable the analysis of multiple parameters from a single sample that is incubated on the array (figure $2 \mathrm{~A}$ ). In reversed-phase protein microarrays, many different samples (cell or tissue lysates) are immobilized in a 
microarray format and are simultaneously analyzed for the presence of a single target protein using a target-specific antibody (figure 2B). Replicates of such protein arrays allow the analysis of hundreds of parameters from minimal amounts of sample. The reversed-phase array format is ideally suited to looking into large sample cohorts. It enables the detection of differentially regulated proteins in healthy and diseased tissue or treated and untreated cells, the identification of disease-specific biomarkers or the analysis of cell signaling networks [Hanash 2003]. Tissue arrays are a special type of reversed-phase microarray and consist of tissue slices that are immobilized on a surface. They are the miniaturized equivalent of classical immunohistochemistry assays and enable the simultaneous analysis of large numbers of tissues with minimum reagent consumption [Kononen et al. 1998]. Currently, forward-phase assays are the most frequently used protein microarray format. Protein arrays are used for detailed expression analysis and for functional protein studies. Forward-phase protein microarray assays are mainly applied for antibody arrays to quantify dozens of analytes that are present in complex samples.

\section{Forward Phase Protein Microarray $(\mu \mathrm{ELISA})$}

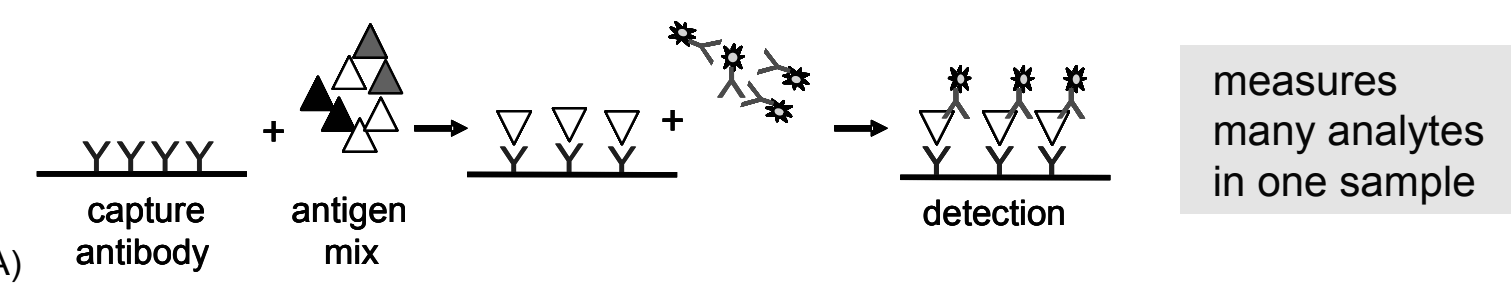

$$
\text { Reverse Phase Protein Microarray (Lysate Array) }
$$

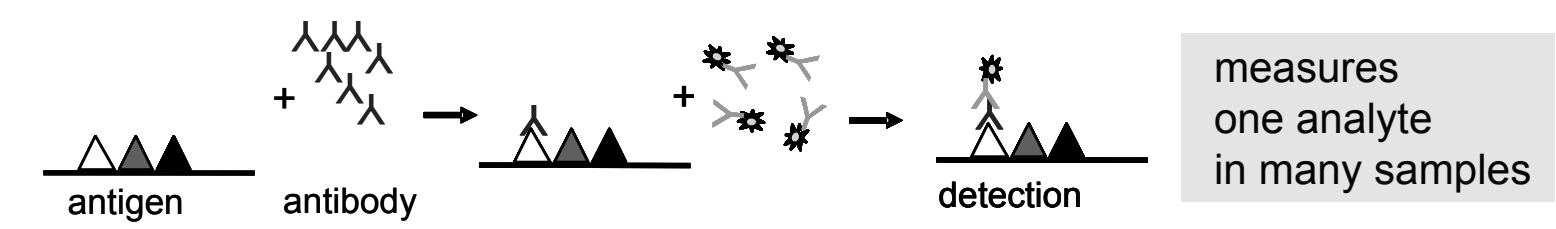

(B) (lysate) mix

Figure 2: Forward and reverse phase protein microarray format. (A) In a forward-phase array format, a large number of immobilized capture molecules enable the analysis of many parameters from a single sample. (B) In a reversedphase array, many samples are immobilized and a highly specific antibody used to analyze the expression of a single parameter in the immobilized samples.

Besides planar microarrays, robust and flexible bead-based assay systems have been developed [Templin 2004]. In planar microarrays, the position within the array enables the identification of an analyte. Bead-based assay systems rely on the use of different bead types that can be distinguished either by a color code, size, or shape. Such bead-based assay systems are also referred to as bead arrays or liquid arrays. The individual bead types are classified according to their internal color code or size in an instrument resembling a flow cytometer. Analytes that are captured on the bead surface are detected by the appropriate reporter molecules. The bead suspension assays are performed using standard laboratory ware (e.g., microtiter plates). Bead-based assays can easily be automated with liquid handling solutions on the market. Sensitivity and accuracy of bead-based systems are similar to those obtained with established ELISA systems involving planar arrays. Some bead-based immunoassays proved to be as sensitive, accurate, and precise as competitive radio immunoassays [Opalka 2003].

Protein microarrays have become well-established research tools in basic and applied research and the first products are already on the market. Despite the huge potential of DNA, proteins are the key players in cellular processes. DNA microarrays only provide a limited amount of information on the status of cells and tissues. Cellular functions depend on protein activity, and proteins are not only regulated by the differential expression of the underlying genes but also by post-translational modifications. Furthermore, protein expression does not generally quantitatively correlate with mRNA expression [Gygi 1999]. Within the last decade, protein microarrays have entered the field of proteomic research demonstrating their huge capacity for identifying and quantifying proteins and for studying the function of proteins from the perspective of the proteome as a whole [Stoll 2004]. Besides their application in proteome-wide research and the determination of the biochemical activities of proteins, protein microarrays are also perfect tools for quantitating subsets of proteins in complex mixtures. This approach is known as focused protein profiling or analytical microarrays. Protein microarray technology has enormous potential for in vitro 
diagnostics. Miniaturized parallelized immunoassays are perfectly suited to generating a maximum of diagnostically relevant information from minute amounts of sample whilst only requiring small amounts of reagent [Hartmann 2008]. A variety of analytical protein microarrays have been developed for different platforms and are entering the diagnostic market. The first planar and bead-based multiplex immunoassays have been cleared by the US FDA or have been CE-marked for use in the EU (figure 3).

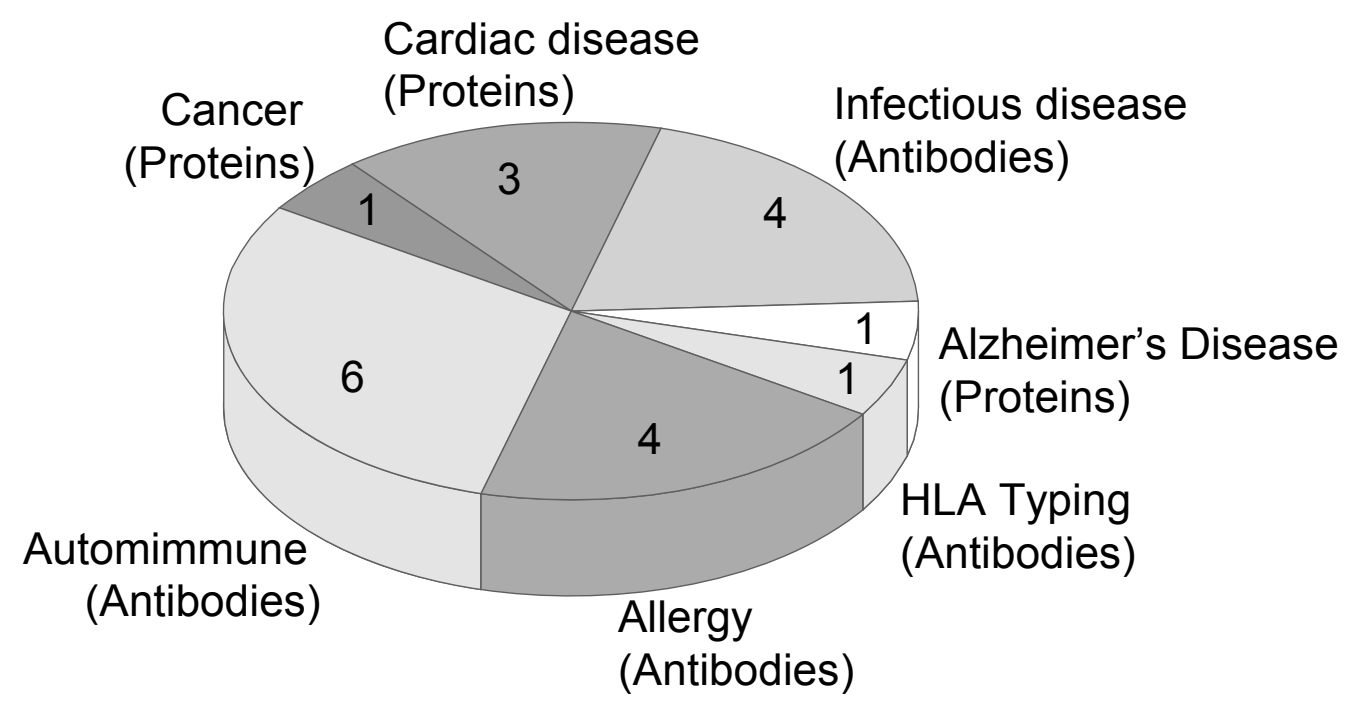

Figure 3: Survey of commercially available multiplexed diagnostics based on protein analysis and the detected analytes. Genetic tests still dominate multiplexed diagnostics, but protein based tests are catching up. So far, predominantly antibodies have been detected in serum from patients suffering from allergies, autoimmune, or infectious diseases. Technically seen, the bead-based Luminex platform is the prevailing technology for multiplexed protein diagnostics (Source: Multiplexed Diagnostics 2008; www.SelectBiosciences.com)

- $\quad$ Ekins RP (1989) J Pharm Biomed Anal 7:155-168

- Hanash S (2003) Nature 422:226-232

- Kononen J, Bubendorf L, Kallioniemi A, Barlund M, Schraml P, Leighton S, Torhorst J, Mihatsch MJ, Sauter G, Kallioniemi OP (1998) Nat Med 4:844-847

- Templin MF, Stoll D, Bachmann J, Joos TO (2004) Comb Chem High Throughput Screen 7:223-229

- Opalka D, Lachman CE, MacMullen SA, Jansen KU, Smith JF, Chirmule N, Esser MT (2003) Clin Diagn Lab Immunol 10:108-115

- Gygi SP, Rochon Y, Franza BR, Aebersold R (1999) Mol Cell Biol 19:1720-1730

- Stoll D, Bachmann J, et al. (2004). Targets 3(1): 24-31

- Hartmann, M., J. Roeraade, et al. (2008). Anal Bioanal Chem.

\footnotetext{
${ }^{1}$ NMI Natural and Medical Sciences Institute at the University of Tübingen, Reutlingen, Germany

${ }^{2}$ School of Chemical Science and Engineering, Department of Analytical Chemistry, Royal Institute of Technology, Stockholm, Sweden
} 